\title{
The Influences of Assembly Position of Annular Slit Restrictor on Dynamic Coefficients of Hydrostatic Guideways
}

\author{
WANG Zhi Wei ${ }^{1, a^{*}}$, ZHA Jun ${ }^{2, b}$ and ZHAO Wan Hua ${ }^{2, c}$ \\ ${ }^{1}$ College of Mechanical and Electronic Engineering, Shandong University of Science and \\ Technology, Qingdao 266590, China \\ ${ }^{2}$ State Key Laboratory for Manufacturing Systems Engineering, Xi'an Jiaotong University, Xi'an \\ 710049, China \\ azwwang@live.com, b zhajun315@126.com, c whzhao@mail.xjtu.edu.cn
}

\begin{abstract}
Keywords: Hydrostatic guideways; Annular slit restrictor; Assembly position; Dynamic coefficients Abstract. Considering the errors of the restrictor and the hole in the form of ellipse, the influences of assembly position of annular slit restrictor and the errors value on stiffness coefficient and damping coefficient of hydrostatic guideways are investigated. It is shown that assembly position of the restrictor have great influence on dynamic coefficients of hydrostatic guideways. Moreover, the greater of the errors value, the greater of influence of assembly position on the hydraulic resistance of the restrictor, stiffness coefficient and damping coefficient. Under the same conditions, the influence of assembly position on damping coefficient is greater than on stiffness coefficient. Improving the machining accuracy of the restrictor or the hole can reduce the influence of assembly position on dynamic performance of hydrostatic guideways. During the process of adjusting the restrictor should take into account the impact of assembly position.
\end{abstract}

\section{Introduction}

Hydrostatic supports have some advantages such as low running friction, high stiffness, high motion accuracy, long service life, superior vibration-resistance and excellent adaptability. So hydrostatic supports are widely used in machine tools, aerospace industry, semiconductor industry, textile industry and measuring instrument[1].

The restrictor is an indispensable part in the hydrostatic supporting system with constant pressure. So the research on the restrictor is always the popular research area. The restrictor used in the hydrostatic supports can be divided into following two categories: restrictor of constant hydraulic resistance and restrictor of variable hydraulic resistance. Mizumoto[2] proposed a hydrostatically-controlled restrictor using a floating ring. The stiffness of bearing with this restrictor can be controlled by setting the design parameters. Meng[3] proposed three new type restrictors, including variable clearance sliding valve, variable clearance sheet and variable diameter capillary. The static stiffness of hydrostatic bearing can be achieve infinite through reasonable design of these kinds of restrictors. However, the dynamic response of hydrostatic support with these kinds of restrictors are generally very slow[4].

In addition, there are a lot of literatures on the research of restrictor of constant hydraulic resistance. Chen[5] studied the influences of capillary restriction parameters on the stability of a Jeffcott rotor-hybrid bearing system. Cheng[6] analyzed the effect of orifice and the eccentricity of the bearing on performance of the hydrostatic bearing. But the restrictor of constant hydraulic resistance (such as capillary and orifice) often suffer from blockage in the use of process.

A new type of annular slit restrictor[7] is used as the research object in this paper. The influences of assembly position of annular slit restrictor and the errors value on stiffness coefficient and damping coefficient of hydrostatic guideways are investigated. 


\section{Influence of assembly position of annular slit restrictor}

Dimensionless stiffness coefficient $(\bar{k})$ and dimensionless damping coefficient $(\bar{c})$ of hydrostatic guideways are obtained by small perturbation method based on Reynolds equation[8]. The flow continuity equation is involved in the small perturbation method. The calculation method of the flow rate of the lubricant through the restrictor is as follows.

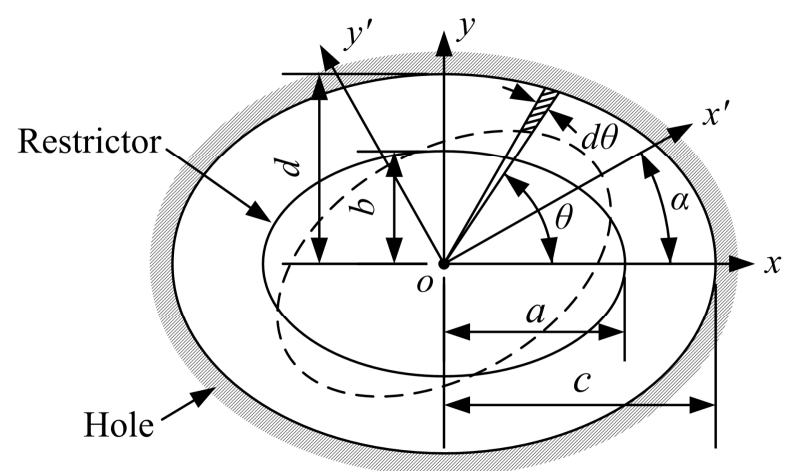

Fig. 1 Schematic diagram of different installation position of annular slit restrictor

Fig. 1 shows the schematic diagram of different installation position of annular slit restrictor. The errors of the restrictor and the hole are assumed as the form of ellipse[9]. Under the condition of no machining errors, the radius of the restrictor and the hole are $r$ and $R$, respectively. $e$ and $E$ are cylindrical errors of the restrictor and the hole, respectively.

$$
\left\{\begin{array}{l}
a=r+e \\
b=r-e \\
c=R+E \\
d=R-E
\end{array} .\right.
$$

Where $a$ and $b$ are the long axis and short axis of the ellipse error of the restrictor. $c$ and $d$ are the long axis and short axis of the ellipse error of the hole.

The flow rate of the lubricant through the restrictor is derived as[10]

$$
Q_{c}=\int_{0}^{2 \pi} \frac{r(\theta) h_{c}^{3}\left(p_{s}-p_{r}\right)}{12 \eta l_{c}} d \theta .
$$

Where $p_{s}$ is the supply pressure, $p_{r}$ is the recess pressure, $h_{c}$ is the thickness of annular slit, the equations of $h_{c}$ is expressed as

$$
\left\{\begin{array}{l}
h_{c}=R(\theta)-r(\theta) \\
R(\theta)=\sqrt{c^{2} \cos ^{2} \theta+d^{2} \sin ^{2} \theta} \\
r(\theta)=\sqrt{a^{2} \cos ^{2}(\theta-\alpha)+b^{2} \sin ^{2}(\theta-\alpha)}
\end{array} .\right.
$$

Where $r(\theta)$ and $R(\theta)$ are polar coordinate equations of the restrictor and the hole, respectively. $\alpha$ is installation angle of the restrictor.

The hydraulic resistance of the annular slit restrictor is obtained as follows,

$$
R_{c}=\frac{p_{s}-p_{r}}{Q_{c}} \text {. }
$$

\section{Results and Discussion}

The variation of hydraulic resistance of the restrictor with $\alpha$ in the range of $0^{\circ} \sim 90^{\circ}$ is shown in Fig. 2 . It is found that the hydraulic resistance of the restrictor decreases with increasing the value of $\alpha$. The influence of $\alpha$ on the hydraulic resistance of the restrictor is greater at a higher value of $e$. In the case of $E=e=10 \mu \mathrm{m}$, the hydraulic resistance of the restrictor is reduced by approximately $48 \%$ with $\alpha$ from $0^{\circ}$ 
to $90^{\circ}$. It is observed that the assembly position of the restrictor has a great influence on the hydraulic resistance of the restrictor, and the influence on the flow rate is also very great.

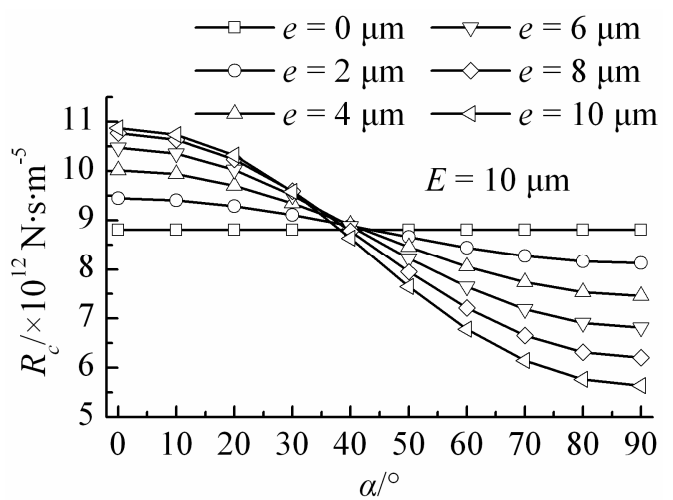

Fig. 2 Hydraulic resistance of the restrictor versus $\alpha$ at different $e$

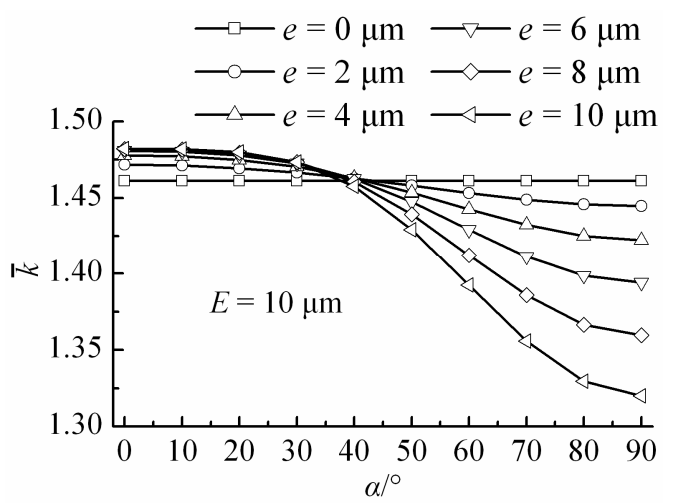

Fig. 3 Dimensionless stiffness coefficient versus $\alpha$ at different $e$

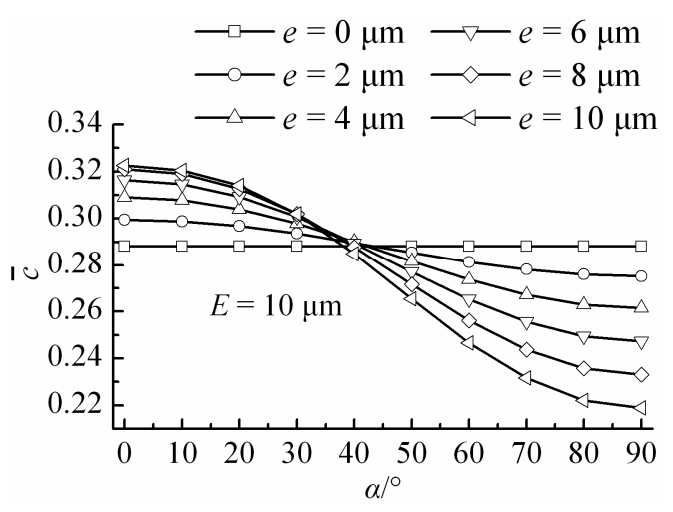

Fig. 4 Dimensionless damping coefficient versus $\alpha$ at different $e$

The variation of dimensionless stiffness coefficient and dimensionless damping coefficient with $\alpha$ at different values of $e$ are shown in Fig. 3 and Fig.4, respectively. Compared with Fig. 2, it is found that the variation trend of dimensionless stiffness coefficient and dimensionless damping coefficient are the same as that of hydraulic resistance. In the case of $E=e=10 \mu \mathrm{m}$, the dimensionless stiffness coefficient is reduced by approximately $11 \%$ and the dimensionless damping coefficient is reduced by approximately $32 \%$ with $\alpha$ from $0^{\circ}$ to $90^{\circ}$. Thus, the influence of the assembly position of the restrictor on the damping coefficient is greater than on the stiffness coefficient under the same conditions. As seen from Fig. 2, Fig. 3 and Fig. 4, the change of $e$ has little effect on the hydraulic resistance, stiffness coefficient and damping coefficient when the vicinity of $\alpha=40^{\circ}$.

Based on the above analyses, it is found that there may be a large difference in the dynamic parameters of hydrostatic guideways in the two assembly process. Thus, improving the machining accuracy of the restrictor or the hole can reduce the influence of assembly position on dynamic performance of hydrostatic guideways. 


\section{Conclusions}

The effects of assembly position of annular slit restrictor on dynamic coefficients of hydrostatic guideways are studied. According to the results obtained, the following conclusions are evident:

1. Assembly position of the restrictor has great influence on dynamic coefficients of hydrostatic guideways. The hydraulic resistance, stiffness coefficient and damping coefficient decreases with increasing installation angle of the restrictor $(\alpha)$. Moreover, the influences of installation angle are greater at a higher value of errors of the restrictor $(e)$.

2. The change of $e$ has little effect on the hydraulic resistance, stiffness coefficient and damping coefficient when the vicinity of $\alpha=40^{\circ}$.

3. The influence of the assembly position of the restrictor on the damping coefficient is greater than on the stiffness coefficient under the same conditions.

4. During the process of adjusting the restrictor should take into account the impact of assembly position. Improving the machining accuracy of the restrictor or the hole can reduce the influence of assembly position on dynamic performance of hydrostatic guideways.

\section{Acknowledgements}

This project is supported by National Natural Science Foundation of China (Grant No. 51505260) and Scientific Research Foundation of Shandong University of Science and Technology for Recruited Talents.

\section{References}

[1] Lin JR. Static and dynamic characteristics of externally pressurized circular step thrust bearings lubricated with couple stress fluids[J]. Tribology International, 1999, 32 (4): 207-216.

[2] H Mizumoto, M Kubo, Y Makimoto et al.. A hydrostatically-controlled restrictor for an infinite stiffness hydrostatic journal bearing[J]. Journal of the Japan Society of Precision Engineering, 1985, 51: 1553-1558.

[3] Xinzhai Meng, Zhaoyan Meng. Analysis and study of new type hydrostatic bearing restrictor with very good restrictive performance[J]. Engineering Science, 2005, 7(03): 49-52. (In Chinese)

[4] Baopei Wang, Peng Zhai, Lei Qin et al.. Research on the dynamic characteristic of hydrostatic bearing[J]. Chinese Hydraulics \& Pneumatics, 2007, (08): 58-61. (In Chinese)

[5] Chen $\mathrm{CH}$, Kang Y, Huang YN, et al. The restrictive effects of capillary compensation on the stability of the Jeffcott rotor-hybrid bearing system[J]. Tribology International, 2002, 35 (12): 849-855.

[6] Chen CH, Kang Y, Huang CC. The influences of orifice restriction and journal eccentricity on the stability of the rigid rotor-hybrid bearing system[J]. Tribology International, 2004, 37 (3): 227-234.

[7] Wanhua Zhao, Yaolong Chen, Zhongyun $\mathrm{He}$ et al.. Annular slit restrictor[p].China 201010102089.4, 2010-09-15. (In Chinese)

[8] Zhiwei Wang, Wanhua Zhao, Bingheng Lu. Influencing factors on dynamic response of hydrostatic guideways[J]. Advanced Materials Research, 2012,418-420: 2095-2101.

[9] Linxiang Cao, Huichong Xie. The measuring accuracies of error separation techniques using the reversal methods[J]. Journal of Northwestern Polytechnical University, 1988, 6(1): 1-10. (In Chinese)

[10] Bassani R, Piccigallo B. Hydrostatic lubrication[M]. Amsterdam: Elsevier Science Ltd, 1992. 